abnormalities, such as del(17p), del(13q14), del(11q22) and +12 in CLL. Yet others like LPL and HCL lack specific cytogenetic aberrations. The recent surge in next generation sequencing technology has shed abundant light on the genetic landscape of SBCLs through characterisation of numerous driver mutations including BRAF (V600E) in HCL and MYD88 (L265P) in LPL, amongst others. These significant advances and panoply of available tests notwithstanding, it is important to appreciate that not all SBCLs need genetic testing for diagnosis, not all harbour their characteristic genetic features and not all are diagnostically specific. This lecture will attempt to review the role of genetic studies in SBCLs, highlighting their advantages and limitations.

\section{B-CELLS BEHAVING BADLY II: A BETTER BASIS TO BEHOLD BELLIGERENCE IN AGGRESSIVE B-CELL LYMPHOMAS}

\author{
Adam Bagg \\ Department of Pathology and Laboratory Medicine, University \\ of Pennsylvania, Philadelphia, PA, USA
}

Aggressive B-cell lymphomas include diffuse large B-cell lymphoma (DLBCL), the most common type of lymphoma encountered in most countries. Despite the single term, DLBCLs are a rather diverse group of lymphomas that can be subclassified using a variety of different approaches, including anatomical site, morphology, immunophenotype, and a number of genetic strategies. Nascent molecular technologies have led to the discovery of many of the genetic events underlying the pathogenesis of this group of diseases. Whether by defining gene signatures that subclassify DLBCL into subgroups based upon cell-of-origin, through dysregulation of key cellular pathways, or specific mutations, we are approaching an era in which personalised diagnostics, prognostication and therapy are imminent. Attempts to develop surrogate immunohistochemical algorithms for some of these genetic phenomena have been variably successful. This lecture will focus on DLBCL and highlight one particular shade of so-called grey zone lymphoma and its relationship to double-hit lymphomas. An attempt will also be made to synthesise the rational use of genetic testing of lymphomas covered in both this and the preceding lecture on small B-cell neoplasms.

\section{SYSTEMIC AND VASCULAR DISEASES IN THE LUNGS}

\section{Andrew G. Nicholson}

Department of Histopathology, Royal Brompton and Harefield NHS Foundation Trust and Imperial College School of Medicine, London, UK

Although many patients with systemic and vascular diseases involving the lungs are diagnosed without recourse to biopsy, pathologists will still be involved on occasion. Indeed, it is often only after multidisciplinary review that the possibility of a systemic cause for the pulmonary symptoms is considered. Clinical correlation is therefore essential, also in relation to the possibility of iatrogenic disease (e.g., drug reactions). In relation to microscopy, for connective tissue disorders in particular, it is important to remember that these diseases can affect all compartments of the lung. As an example, rheumatoid disease is not only associated with patterns of interstitial pneumonia, but also, rheumatoid nodules, apical fibrosis, airways disease (follicular bronchitis, bronchiectasis, bronchocentric granulomatosis, constrictive obliterative bronchiolitis), pulmonary hypertension, and rarely development of malignancies in the lung. Also, it is important to remember that one pattern of disease may progress to another, dependent on the disease process, so longitudinal behaviour is an additional key factor when reviewing samples. In relation to pulmonary vascular disease, excluding vascular tumours, there also remains a minority of cases where biopsy still plays a role (e.g., veno-occlusive disease, embolic disease). Typically, a surgical lung biopsy is required, although transbronchial biopsy may rarely yield information.

\section{CUTANEOUS MANIFESTATIONS OF SYSTEMIC DISEASE}

Rooshdiya Z. Karim $^{1,2}$

${ }^{1}$ Department of Tissue Pathology and Diagnostic Oncology, Royal Prince Alfred Hospital, Sydney, and ${ }^{2}$ University of Sydney, Faculty of Medicine, Central Clinical School, Sydney, NSW, Australia

The cutaneous manifestations of systemic diseases are vast and varied. The skin is our largest organ and can act as a mirror to reflect internal pathology including infections; immune, autoimmune and rheumatic diseases; genetic, metabolic, endocrine and nutritional states; internal malignancies; cancer syndromes; haematological diseases; and drug effects.

The cutaneous manifestations may provide the prompt to search for the underlying systemic disease, may assist in classification of the systemic disease and can be the main symptoms of distress for the patient; hence, accurate pathological diagnosis with clinical correlation is critical.

\section{WHAT DO I NEED TO KNOW ABOUT SOMATIC MUTATION TESTING AS A TISSUE PATHOLOGIST - A SURVIVAL GUIDE?}

Sandra O'Toole

Molecular Diagnostic Oncology, Department of Tissue Pathology and Diagnostic Oncology, Royal Prince Alfred Hospital, and University of Sydney, Sydney, NSW, Australia

New technologies have enabled high throughput and detailed analysis of the genetic changes in a range of tumours with increasing recognition that these may assist in the diagnosis and selection of treatment.

In subsets of patients with advanced non-small cell lung cancer, melanoma and colorectal carcinoma, genetic analysis and matched targeted therapies have become standard of care. In lymphomas, detection of MYC gene rearrangement is increasingly used to assist in treatment selection. Characteristic gene rearrangements have been identified in up to 53 different bone and soft tissue tumours as well as in a range of head and neck and renal tumours among others.

Tissue pathologists need to develop a working understanding of the principles of techniques, their advantages and disadvantages, and critically, how to integrate molecular results into the diagnostic process. This lecture will outline principles of molecular assays and technologies, the key indications for their use, and 